\title{
ROCK1 Inhibitor Abrogates Chronic Rejection in Rat Cardiac Model System
}

\author{
Li Zhang $^{1,2,3}$, Malgorzata Kloc ${ }^{1,2 *}$, Neelam Tejpal ${ }^{1,2}$, Junping You ${ }^{1,2}$, Andrea M. Cordero-Reyes ${ }^{4}$, \\ Keith A. Youker ${ }^{4}$, Rafik M. Ghobrial ${ }^{1,2^{*}}$ \\ ${ }^{1}$ Department of Surgery, The Methodist Hospital, Houston, USA \\ ${ }^{2}$ The Methodist Hospital Research Institute, Houston, USA \\ ${ }^{3}$ The Department of Transplantation, The First Central Clinical College of Tianjin Medical University, Tianjin, China \\ ${ }^{4}$ The Methodist DeBakey Heart \& Vascular Center, Houston, USA \\ Email: *mkloc@tmhs.org, *RMGhobrial@tmhs.org
}

Received September 20, 2012; revised October 29, 2012; accepted November 8, 2012

\begin{abstract}
Background: We have shown previously that the abrogation of acute and chronic rejection of rat cardiac allografts occurs through the down-regulation of RhoA pathway and involves the changes in RhoA kinase (ROCK)-dependent actin cytoskeleton and $\mathrm{T}$ cell motility. Here we studied the ability of the Y-27632, a highly selective inhibitor of Rho-associated protein kinase p160ROCK (ROCK1), to abrogate chronic rejection of the allograft and influence T cell infiltration. Methods: Heterotopic cardiac transplants were performed between donor Wistar Furth (WF) and ACI recipient rats. Controls received sub-therapeutic dose of cyclosporine (CsA, $10 \mathrm{mg} / \mathrm{kg}$ ) for 3 days or 7 days therapeutic dose of cyclosporine. The experimental groups of ACI recipients received one preoperative dose of the Y-27632 inhibitor (2 mg/kg, gavage feed) in conjunction with the sub-therapeutic dose of CsA for 3 days or inhibitor alone for 7 days. The cardiac grafts were harvested at 100 days of post-transplantation for histological and immunohistochemical assessment of chronic rejection, vascular sclerosis, and infiltration by different T cell subtypes. Results: Cardiac allografts from recipients exposed to Y-27632 inhibitor in conjunction with sub-therapeutic dose of CsA showed drastically reduced vascular sclerosis, minimal myocardial total cellular infiltration, and were selectively infiltrated with Foxp3 $^{+} \mathrm{T}$ regulatory (Treg) cells. Conclusions: Our novel finding that a single dose of the ROCK1 inhibitor Y-27632 attenuates chronic rejection in rat cardiac model system by promoting development of Treg cells warrants its potential as a novel therapeutic agent specific for the inhibition of chronic rejection.
\end{abstract}

Keywords: Chronic Rejection; Transplantation; ROCK1; RhoA; T Cell

\section{Introduction}

One of the most challenging and still unresolved problems in organ transplantation is the phenomenon of chronic rejection that slowly damages and eventually destroys transplanted organs [1-5]. At present, none of the available immunosuppressive drugs, while being effective in abrogating acute rejection, is able to inhibit chronic rejection [3-5]. In order to study the mechanisms and molecular pathways responsible for the chronic rejection and to identify potential molecular targets and therapeutic agents we previously developed the rat cardiac allograft model system in which the peri-operative treatment of recipients with an allochimeric MHC I induced indefinite survival and abrogated acute and chronic rejection of the allograft [6-9]. In this model system the dominant immunogenic epitopes were identified

"Corresponding author. within the hypervariable regions of the $\alpha 1$ domain of RT1.A ${ }^{\mathrm{a}}$, and RT1.A $\mathrm{A}^{\mathrm{u}}$. The peri-transplant, portal vein delivery of MHC class I allochimeric protein, which displays the $\alpha 1$-helical epitopes of RT1.A ${ }^{\mathrm{u}}$ on recipent-type $\mathrm{RT} 1^{\mathrm{a}}$ sequences, induced donor-specific tolerance of Wistar Furth (WF, RT1 ${ }^{\mathrm{u}}$ ) hearts in ACI $\left(\mathrm{RT}^{\mathrm{a}}\right)$ hosts in the presence of subtherapeutic cyclosporine (CsA, 10 $\mathrm{mg} / \mathrm{kg} / \mathrm{d} \times 3$ days). Cytokines analysis of long-term allografts revealed selective upregulation of IL-10, but marked inhibition of IL-2, IFN- $\gamma$, and IL-4. Furthermore, long-term allografts in allochimeric-conditioned hosts exhibited moderate B cell infiltration, limited monocyte and absent $\mathrm{CD}^{+}$cells [6-9]. In addition, our adoptive transfer studies showed that the secondary cardiac grafts from recipients exposed to allochimeric MHC I-conditioned splenic total $\mathrm{T}$ cells or $\mathrm{CD} 4^{+} \mathrm{T}$ cells showed significantly reduced neointimal index (NI) and apoptosis, and were selectively infiltrated with $\mathrm{CD}^{+} \mathrm{Foxp}^{+}$( $\mathrm{T}$ regulatory, Treg) cells [10]. While the allochimeric therapy is 
clinically applicable, it may be faced with the complexity of the MHC in humans, definition of appropriate sequences and the requirements of rapid synthesis at the time of transplantation. We have therefore focused on utilizing the ability of allochimeric molecules to abrogate chronic rejection as an investigative tool to identify target molecules critical for the inhibition of chronic rejection. Targeting of such molecules would provide a clear path to clinical utility. Recently, we showed that allochimeric MHC I treatment induced down-regulation of RhoA pathway components (RhoA and ROCK-1) involved in actin cytoskeleton organization and cell motility, caused the inhibition of T cell migration into the graft [11-14] and restriction of the TCR repertoire [15]. Proper actin cytoskeleton architecture and dynamics, indispensable for events in the immunological response such as $\mathrm{T}$ cell interaction with antigen presenting cells, activation, migration and redistribution of $\mathrm{T}$ cell receptors [16-23] are regulated by proteins belonging to the superfamily of small GTP-binding proteins, such as RhoA GTPase and its downstream target, the RhoA kinase (ROCK) [24-31]. Our finding that in rat cardiac allograft model system the immunosuppressive and anti-chronic rejection activity of the allochimeric molecule was achieved via down regulation of the RhoA pathway- and cytoskeleton-dependent $\mathrm{T}$ cell functions $[11,13,14]$ suggested that a RhoA pathway inhibitor(s) could be a potential therapeutic agent(s) for the inhibition of chronic rejection of the grafts. Thus, we tested the ability of the Y-27632, a highly selective, commercially available, inhibitor of Rho-associated protein kinase p160ROCK (ROCK1) [32,33], to abrogate chronic rejection in rat cardiac allografts.

\section{Material and Methods}

\subsection{Animals}

Adult male inbred Wistar Furth (WF; RT1.A ${ }^{\mathrm{u}}$ ) and ACI $\left(\right.$ RT1.A $\left.{ }^{a}\right)$ rats were purchased from Harlan Sprague Dawley (Indianapolis, IN) and housed in standard rat cages. Heterotopic cardiac transplants were placed intraabdominally as described previously [6-15]. Transplantation was performed as follows: 1) transplantation control group that received no treatment; 2) transplantation in the presence of high dose cyclosporine (CsA) delivered by gavage feed $(10 \mathrm{mg} / \mathrm{kg}$, day 0 - 6); 3) transplantation in the presence of sub-therapeutic dose of CsA delivered by gavage (10 mg/kg, day 0 - 2); 4) transplantation in the presence of Y-27632 inhibitor (Fisher Scientific, $2 \mathrm{mg} / \mathrm{kg}$, day 0 - 6) delivered by gavage; and 5) transplantation in the presence of sub-therapeutic dose of CsA $(10 \mathrm{mg} / \mathrm{kg}$, day 0 - 2) in conjunction with Y-27632 inhibitor (2 $\mathrm{mg} / \mathrm{kg}$ ) which was gavage fed as a single dose before the transplantation. Graft survival was assessed by abdominal palpation for a heartbeat daily for 2 weeks and then once weekly until no heartbeat was palpable. Three to five animals in each experimental group were assessed for graft survival, as described above, and euthanized at 100 days or when heartbeat had ceased. Animals were housed postoperatively according to standard protocols and all experiments were conducted under the Methodist Hospital Research Institute's animal care guidelines and the NIH standards set forth in the "Guide for the Care and Use of Laboratory Animals” (DHHS publication No. (NIH) 85-23 Revised 1985).

\subsection{Histopathology and Immunostaining}

Rat hearts were fixed in formalin, embedded in paraffin and $5 \mu \mathrm{M}$ sections were cut at the mid-heart region. Sections were stained with Hematoxylin \& Eosin (H\&E), Trichrome or Verhoeff-van Gieson (VVG) using standard protocols. H\&E staining was used to examine heart global architectural integrity and inflammation.

Immunohistochemistry was performed using following primary antibodies: anti-IgG (\#SC-2041, Santa Cruz Biotechnology, Santa Cruz, CA), anti-IgM (\#31476, Fisher Thermo Scientific, Waltham, MA), anti-Foxp3 (\# 14-4774-80, eBioscience, San Diego, CA, USA), antiIL2ra (CD25, \# MA1-70019, Fisher Thermo Scientific, Waltham, MA), anti-ICAM (\#554967, BD Pharmingen, San Diego, CA, USA), anti-LFA-1 (\# 559979, BD Pharmingen, San Diego CA, USA), anti-CD4 (\#MA1-81588, Fisher Thermo Scientific, Waltham, MA) and anti-CD3 antibody (\#MA1-81580, Fisher Thermo Scientific, Waltham, MA). The secondary antibodies were conjugated with horseradish peroxidase and DAB staining was performed. Results were reported as negative (0), low positive (1), moderate positive (2) and high positive (3) staining. Averages were calculated by assigning the following numerical values: $(0)=0$ cells, $(1)=1-2$, $(2)=3$ 10 and $(3)=11+$ cells per low magnification filed. A blind observer performed analysis of rat hearts, and the results were de-coded after the completion of the analysis.

\subsection{Statistical Analysis}

Collagen deposition was quantitated from Trichrome stained slides using Image-Pro Plus and is presented as \% of stained area/total area. VVG staining was used to analyze the neointima and is presented as the neointimal index $(\mathrm{NI}=($ intimal area $) /($ luminal area + intimal area $) \times$ 100). Three to five hearts from each experimental group were analyzed. 10 - 8 vessels from each heart section were analyzed. To avoid erroneously high indices, only vessels of a minimum size of $85 \mu \mathrm{M}$ or larger diameter were analyzed. A cardiac pathologist blindly reviewed the slides in parallel and confirmed the findings using standard pathological based criteria for neointimal involvement. Standard deviation and $\mathrm{P}$ values were calculated in Excel. 


\section{Results}

\subsection{RhoA Pathway Inhibitor Treatment Abrogates Chronic Rejection of the Graft}

We established that the pre-transplantation gavage delivery of a single dose (2 mg/kg) of Y-27632 inhibitor in conjunction with sub-therapeutic dose of CsA $(10 \mathrm{mg} / \mathrm{kg}$, day 0 - 2), seven-day treatment with the Y-27632 inhibitor alone (gavage delivery, $2 \mathrm{mg} / \mathrm{kg}$, day 0 - 6) or therapeutic dose of CsA alone (day 0 - 6, $10 \mathrm{mg} / \mathrm{kg}$ ) resulted in long term (>100 days) graft survival and, as established previously, the untreated animals and recipients treated with sub-therapeutic dose of CsA (day 0 - 2, $10 \mathrm{mg} / \mathrm{kg}$ ) promptly rejected the allograft [6-10]. Although these data indicated that allograft survival was prolonged in recipients that received therapeutic dose of CsA or a seven-day treatment with the Y-27632 inhibitor alone or a single dose of Y-27632 inhibitor in conjunction with sub-therapeutic dose of CsA, we found that the abrogation of chronic rejection occurred exclusively in the single dose of Y-27632 inhibitor in conjunction with subtherapeutic dose of CsA-treated group. Comprehensive analysis of the allograft tissue and an assessment of cardiac vasculopathy showed dramatic differences in the degree of chronic rejection between allografts from recipients that had received Y-27632 inhibitor in conjunction with sub-therapeutic dose of CsA and those treated with inhibitor alone or CsA alone (Table 1). There was a significant reduction of the neointimal hyperplasia (NI) in the allograft from the recipients that received Y-27632 inhibitor in conjunction with sub-therapeutic dose of CsA in comparison to those treated with therapeutic dose of CsA or inhibitor alone (Table 1, Figure 1; 13.9 vs. 45.1 and 76.9 respectively, p < 0.0002). Similar trend persisted in the collagen deposition present in the allografts (Table 1, Figure 1; 20.5\% vs. $22.6 \%$ and $80.2 \%$ respectively).

\subsection{RhoA Pathway Inhibitor Treatment Inhibits Cellular Infiltration and Promotes Development of T Regulatory Cells}

Histochemical analysis of H\&E stained sections demonstrated minimal myocardial cellular infiltrates in the allograft from the recipients that received Y-27632 inhibitor in conjunction with sub-therapeutic dose of CsA in comparison to those treated with CsA or inhibitor alone (Figure 2). Immunostaining with antibodies against IgG, IgM, integrin CD11a and its ligand, the intercellular adhesion molecule 1 (ICAM-1 also known as a cluster of differentiation 54 CD54), which is a cell surface glycoprotein stabilizing cell-cell interactions and facilitating leukocyte endothelial transmigration), and IL2 receptor $\alpha$ subunit CD25, which is expressed on antigen activated $\mathrm{T}$
Table 1. Chronic rejection score.

\begin{tabular}{ccccc}
\hline Treatment & NI & $\begin{array}{c}\text { Affected } \\
\text { vessels }\end{array}$ & $\begin{array}{c}\text { Unaffected } \\
\text { vessels }\end{array}$ & $\begin{array}{c}\text { Collagen } \\
\text { content }\end{array}$ \\
\hline Y-27632 + CsA 3 days & $13.9 \pm 19.7$ & $29 \%$ & $71 \%$ & $20.5 \%$ \\
Y-27632 7 days & $76.9 \pm 22.8$ & $77 \%$ & $23 \%$ & $80.2 \%$ \\
CsA 7 days & $45.1 \pm 37.5$ & $60 \%$ & $40 \%$ & $22.6 \%$ \\
\hline
\end{tabular}
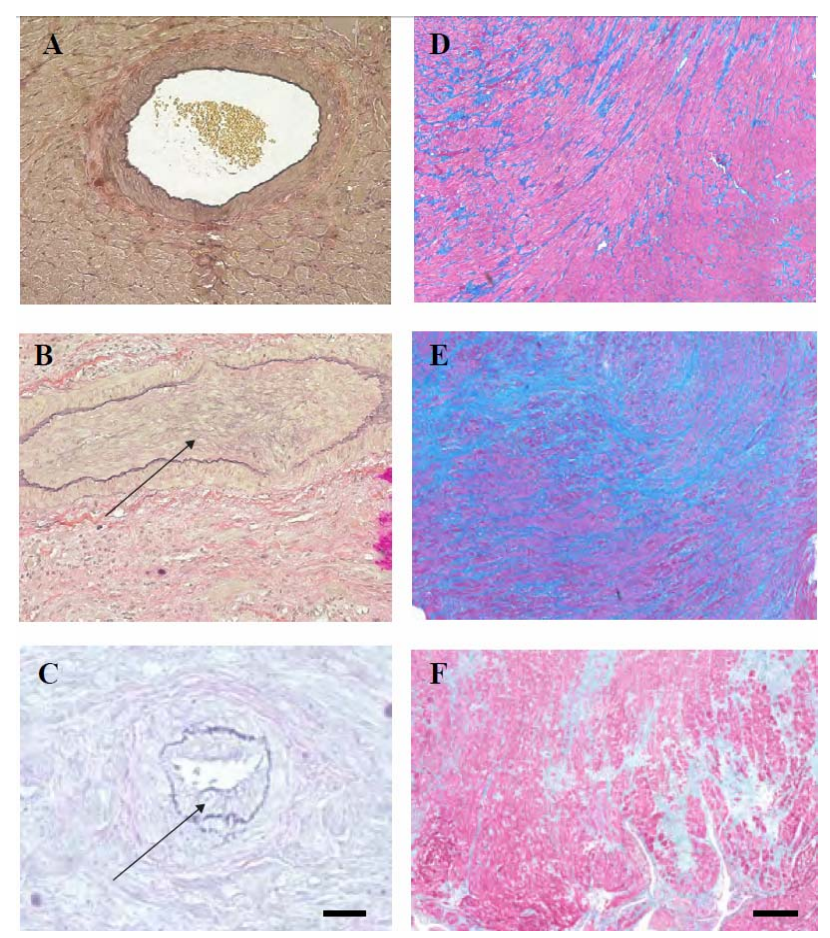

Figure 1. Cardiac allografts' vasculopathy and collagen deposition (A-C) Verhoeff-Van Gieson (VVG) staining, and (D-F) Trichrome staining of transplanted hearts at 100 days post-transplantation. Hearts from the recipients treated with one preoperative dose of the Y-27632 inhibitor in conjunction with the sub-therapeutic dose of CsA show low neointimal hyperplasia (NI) (A) and collagen deposition (D) when compared to the harts from recipients treated with theY-27632 inhibitor alone (B and E) or therapeutic dose of CsA alone ( $C$ and F). Arrows in (B) and (C) point to the neointima. Bar is equal to $50 \mu \mathrm{m}$.

cells did not show any significant difference between experimental groups (Table 2). However, the results of immunostaining for the presence of different subpopulation of $\mathrm{T}$ cells within the grafts showed a stark increase in the number of cells expressing Treg cell marker Foxp $3^{+}$ within the grafts of recipients treated with Y-27632 inhibitor in conjunction with sub-therapeutic dose of CsA, in comparison to the grafts from CsA or Y-27632 alonetreated groups (Table 2). All these observations indicate the Y-27632 inhibitor-treatment attenuates chronic rejection of the graft through the inhibition of graft infiltration and promotion of development of Treg cells. 


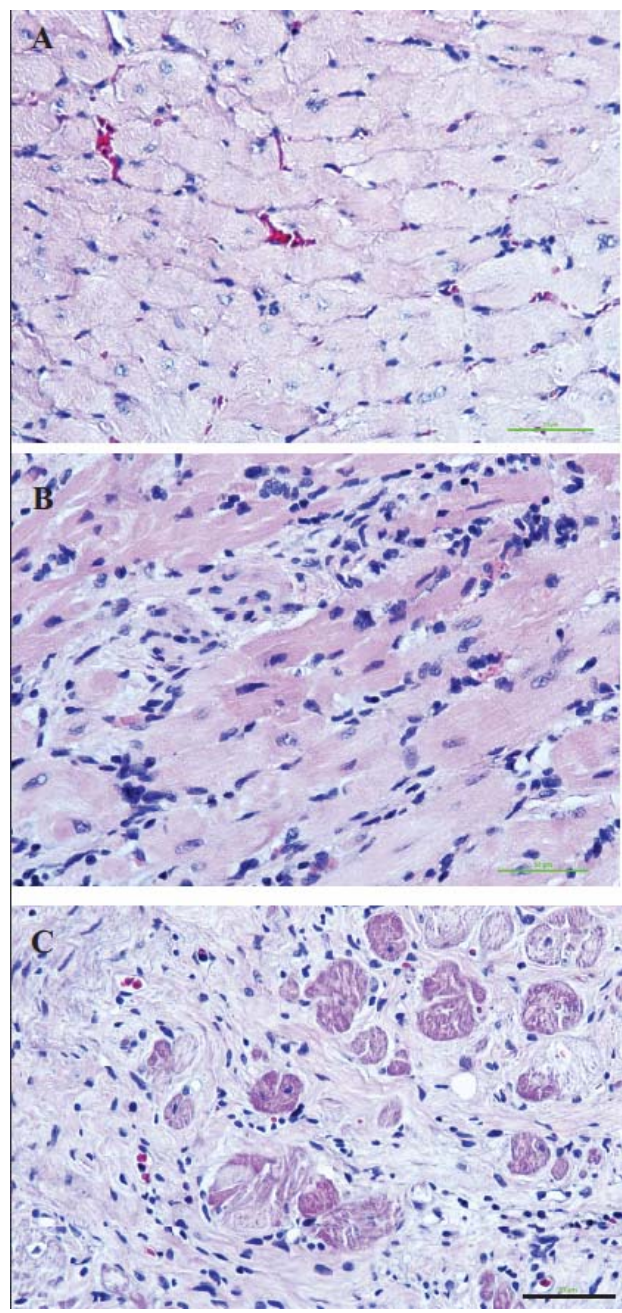

Figure 2. Tissue integrity and cellular infiltration of cardiac allografts Hematoxylin \& Eosin (H\&E) staining of transplanted hearts at 100 days post-transplantation show wellpreserved tissue integrity, low degree of fibrosis and low cellular infiltration in the grafts from the recipients treated with one preoperative dose of the $\mathrm{Y}-27632$ inhibitor in conjunction with the sub-therapeutic dose of CsA (A) when compared to the harts from recipients treated with the $\mathrm{Y}$ 27632 inhibitor alone (B) or therapeutic dose of CsA alone (C). Bar is equal to $50 \mathrm{\mu m}$.

Table 2. Cellular infiltration/immunostaining.

\begin{tabular}{|c|c|c|c|c|c|c|}
\hline Treatment & CD11a & ICAM-1 & CD25 & Foxp3 & CD4 & CD3 \\
\hline $\begin{array}{l}\text { Y-27632 + } \\
\text { CsA } 3 \text { days }\end{array}$ & moderate & low & low & high & \multicolumn{2}{|c|}{ moderate moderate } \\
\hline $\begin{array}{l}\text { Y-27632 } \\
7 \text { days }\end{array}$ & moderate & high & high & moderate & high & high \\
\hline CsA 7 days & moderate & none & low & moderate & low & low \\
\hline
\end{tabular}

\section{Discussion}

In spite of the decades of extensive studies on the phenomenon of allograft rejection, the understanding of the mechanisms underlying the development of chronic re- jection and the ability to inhibit this process are far from being successful. Our previous studies using rat cardiac allograft model system indicated that the abrogation of chronic rejection involves inhibition of $\mathrm{T}$ cell migration into the graft, promotes development of Tregs, and, at the molecular level, correlates with a down regulation of RhoA pathway components and dis-regulation of its actin cytoskeleton targets [10-14]. Here we showed that the treatment with the Y-27632, a highly selective inhibitor of the RhoA protein kinase ROCK1 abrogates chronic rejection of the rat cardiac allografts. Studies on human Jurkat $\mathrm{T}$ cell line transfected with a dominant negative, kinase-defective mutant of Rho kinase diminished Jurkat cell proliferation, attenuated the expression of $\mathrm{T}$ cell activation cytokines, blocked actomyosin polymerization, and prevented aggregation of the TCR/CD3 complex with lipid rafts [32]. The same study showed that in the C57BL/6 (H-2 $\left.{ }^{\mathrm{b}}\right)$ to (DBA/2 x BALB/c)F1 (H-2 ${ }^{\mathrm{d}}$ ) mouse model the treatment with Y-27632 inhibitor at $20 \mathrm{mg} / \mathrm{kg}$ by s.c. continous infusion with an osmotic pump beginning 2 days before transplant and continuing for 26 days, prolonged graft survival and diminished intragraft expression of cytokine mRNAs at 7 days post-transplantation [32]. Another study on the BALB/c $\left(\mathrm{H}-2^{\mathrm{d}}\right)$ to $\mathrm{C} 3 \mathrm{H} /$ $\mathrm{He}\left(\mathrm{H}-2^{\mathrm{k}}\right)$ mouse model, showed that the cardiac allografts from the recipients continuously receiving $\mathrm{Y}$ 27632 orally at $2 \mathrm{mg} / \mathrm{kg} /$ day (commencing 1 day before cardiac transplantation until 100 days) had low expression of myocardial ICAM-1 and VCAM-1 at Day 7 and no apparent thickening of vascular intima at 100 days [33]. In cited above studies very high doses (2 mg - 20 $\mathrm{mg} / \mathrm{kg}$ ) of Y-27632 inhibitor were delivered continuously through the part or whole duration of the experiment. In contrast, our study is the first to show that a single preoperative $2 \mathrm{mg} / \mathrm{kg}$ dose of orally administered Y-27632 in conjunction with sub-therapeutic dose of CsA is able to inhibit chronic rejection of the cardiac allografts.

\section{Conclusion}

Our novel finding that a single pre-operative dose of the RhoA pathway inhibitor Y-27632 attenuates chronic rejection in rat cardiac model system by promoting development of Treg cells warrants its potential as a novel therapeutic agent specific for the inhibition of chronic rejection.

\section{Acknowledgements}

NIH Grant RO1 AI49945 to R. M. Ghobrial, Li Zhang was supported by Dr. Zhongyang Shen and The Department of Transplantation, The First Central Clinical College of Tianjin Medical University, Tianjin 300070, China. 


\section{REFERENCES}

[1] H. L. Trivedi, "Immunobiology of Rejection and Adaptation,” Transplantation Proceedings, Vol. 39, No. 3, 2007, pp. 647-652. doi:10.1016/j.transproceed.2007.01.047

[2] G. W. Butcher and J. C. Howard, "Genetic Control of Transplant Rejection,” Transplantation, Vol. 34, No. 4, 1982, pp. 161-166. doi:10.1097/00007890-198210000-00001

[3] D. L. Dunn, "Problems Related to Immunosuppression. Infection and Malignancy Occurring after Solid Organ Transplantation," Critical Care Clinics, Vol. 6, 1990, pp. 955-977.

[4] A. I. Colovai, E. Renna-Molajoni, R. Cortesini and N. Suciu-Foca, "New Approaches to Specific Immunomodulation in Transplantation," International Reviews of Immunology, Vol. 13, No. 3, 1996, pp. 161-172. doi:10.3109/08830189609061745

[5] O. Joffre, T. Santolaria, D. Calise, T. Al Saati, D. Hudrisier, P. Romagnoli, et al., "Prevention of Acute and Chronic Allograft Rejection with $\mathrm{CD}^{+} \mathrm{CD} 25^{+} \mathrm{Foxp}^{+}$ Regulatory T Lymphocytes,” Nature Medicine, Vol. 14, 2008, pp. 88-92. doi:10.1038/nm1688

[6] R. M. Ghobrial, T. Hamashima, M. Wang, S. M. Stepkowski and B. D. Kahan, "Induction of Transplantation Tolerance by Chimeric Donor/Recipient Class I RT1.Aa Molecules," Transplantation, Vol. 62, No. 7, 1996, pp. 1002-1010. doi:10.1097/00007890-199610150-00020

[7] N. Semiletova, X.-D. Shen, M. C. Fishbein, F. Gao, S. J. Slomowitz, Q. Jiao, et al., "Posttransplant Administration of Allochimeric Major Histocompatibility Complex ClassI-Molecules Induces True Transplantation Tolerance,” Transplantation, Vol. 75, No. 4, 2003, pp. 550-553. doi:10.1097/01.TP.0000046942.02001.26

[8] N. V. Semiletova, X.-D. Shen, B. Baibakov, D. M. Feldman, K. Mukherjee, J. M. Frank, et al., "Inhibition of Chronic Rejection by Antibody Induced Vascular Accommodation in Fully Allogeneic Heart Allografts," Transplantation, Vol. 80, No. 11, 2005, pp. 1535-1340. doi:10.1097/01.tp.0000188952.10692.18

[9] J. S. Singer, A. Mhoyan, M. C. Fishbein, X.-D. Shen, F. Gao, D. Zhao, et al., "Allochimeric Class I MHC Molecules Prevent Chronic Rejection and Attenuate Alloantibody Responses,” Transplantation, Vol. 72, No. 8, 2001, pp. 1408-1416. doi:10.1097/00007890-200110270-00014

[10] T. Skelton, A. M. Cordero, N. J. Nelles, M. Ganachari, J. Sidhu, N. Tejpal, et al., "Allochimeric MHC I-Conditioned T Cells Attenuate Chronic Rejection in Rat Cardiac Model System," Journal of Clinical Cell Immunology, Vol. 2, 2011, p. 108.

[11] W. Lisik, N. Tejpal, Y. Gong, T. S. Skelton, M. Ganachari, E. G. Bremer, et al., "Down Regulation of Genes Involved in T Cell Polarity and Motility during the Induction of Heart Allograft Tolerance by Allochimeric MHC I,” PLoS One, Vol. 4, 2009, Article ID: e8020. doi:10.1371/journal.pone.0008020

[12] T. S. Skelton, N. Tejpal, Y. Gong, J. Z. Kubiak, M. Kloc and R. M. Ghobrial, "Allochimeric Molecules and Mechanisms in Abrogation of Cardiac Allograft Rejection,”
Journal of Heart Lung Transplantation, Vol. 31, No. 1, 2012, pp. 73-84. doi:10.1016/j.healun.2011.01.715

[13] T. S. Skelton, N. Tejpal, Y. Gong, M. Kloc and R. M. Ghobrial, "Downregulation of RhoA and Changes in T Cell Cytoskeleton Correlate with the Abrogation of Allograft Rejection,” Transplantation Immunology, Vol. 23, No. 4, 2010, pp. 185-193. doi:10.1016/j.trim.2010.06.009

[14] W. Lisik, Y. Gong, N. Tejpal, T. S. Skelton, E. G. Bremer, M. Kloc, et al., "Intragraft Gene Expression Profile Associated with the Induction of Tolerance by Allochimeric MHC I in the Rat Heart Transplantation Model," Genesis, Vol. 48, No. 1, 2010, pp. 8-19.

[15] D. Liu, X. D. Shen, Y. Zhai, W. Lam, J. Liao, R. W. Busuttil and R. M. Ghobrial, "Intragraft Selection of the T Cell Receptor Repertoire by Class I MHC Sequences in Tolerant Recipients,” PLoS One, Vol. 4, 2009, Article ID: e6076. doi:10.1371/journal.pone.0006076

[16] J. K. Burkhardt, E. Carrizosa and M. H. Shaffer, "The Actin Cytoskeleton in T Cell Activation," Annual Reviews of Immunology, Vol. 26, 2008, pp. 233-259. doi:10.1146/annurev.immunol.26.021607.090347

[17] M. M. Al-Alwan, R. S. Liwski, S. M. M. Haeryfar, W. H. Baldridge, D. W. Hoskin, G. Rowden and K. A. West, "Cutting Edge: Dendritic Cell Actin Cytoskeletal Polarization during Immunological Synapse Formation Is Highly Antigen-Dependent,” Journal of Immunology, Vol. 171, No. 9, 2003, pp. 4479-4483.

[18] C. Brossard, V. Feuillet, A. Schmitt, C. Randriamampita, M. Romao and G. Raposo, "Trautmann, A. Multifocal Structure of the T Cell-Dendritic Cell Synapse,” European Journal of Immunology, Vol. 35, No. 6, 2005, pp. 17411753. doi:10.1002/eji.200425857

[19] M. L. Dustin, "Cell Adhesion Molecules and Actin Cytoskeleton at Immune Synapses and Kinapses," Current Opinion in Cell Biology, Vol. 19, No. 5, 2007, pp. 529533. doi:10.1016/j.ceb.2007.08.003

[20] M. L. Dustin and J. A. Cooper, “The Immunological Synapse and the Actin Cytoskeleton: Molecular Hardware for T Cell Signaling," Nature Immunology, Vol. 1, No. 1, 2000, pp. 23-29. doi:10.1038/76877

[21] Y. Huang and J. K. Burkhardt, "T-Cell-Receptor-Dependent Actin Regulatory Mechanisms,” Journal of Cell Sciences, Vol. 120, No. 5, 2007, pp. 723-730. doi:10.1242/jcs.000786

[22] D. Sancho, M. Vicente-Manzanares, M. Mittelbrunn, M. C. Montoya, M. Gordon-Alonso, J. M. Sarrador and F. Sanchez-Madrid, "Regulation of Microtubule-Organizing Center Orientation and Actomyosin Cytoskeleton Rearrangement during Immune Interactions,” Immunological Reviews, Vol. 189, No. 1, 2002, pp. 84-97. doi:10.1034/j.1600-065X.2002.18908.X

[23] M. Vicente-Manzanares and F. Sánchez-Madrid, "Role of the Cytoskeleton during Leukocyte Responses," National Reviews of Immunology, Vol. 4, No. 2, 2004, pp. 110-122. doi:10.1038/nri1268

[24] I. Corre, M. Gomez, S. Vielkind and D. A. Cantrell, “Analysis of Thymocyte Development Reveals That the GTPase RhoA Is a Positive Regulator of T Cell Receptor Responses in Vivo," Journal of Experimental Medicine, 
Vol. 194, No. 7, 2001, pp. 903-913. doi:10.1084/jem.194.7.903

[25] M. Vicente-Manzanares, M. Rey, M. Pérez-Martı́nez, M. Ya'ñez-Mo', D. Sancho, J. R. Cabrero, O. Barreiro, H. de la Fuente, K. Itohand F. Sa'nchez-Madrid, "The RhoA Effector mDia Is Induced during $\mathrm{T}$ Cell Activation and Regulates Actin Polymerization and Cell Migration in T Lymphocytes,” Journal of Immunology, Vol. 171, No. 2, 2003, pp. 1023-1034.

[26] Y. Xu, J. Li, G. D. Ferguson, F. Mercurio, G. Khambatta, L. Morrison, A. Lopez-Girona, L. G. Corral, D. R. Webb, B. L. Bennett and W. Xie, "Immunomodulatory Drugs Reorganize Cytoskeleton by Modulating Rho GTPases,” Blood, Vol. 114, No. 2, 2009, pp. 338-345. doi:10.1182/blood-2009-02-200543

[27] A. B. Pernis, "Rho GTPase-Mediated Pathways in Mature $\mathrm{CD}^{+}$T Cells," Autoimmunity Reviews, Vol. 8, No. 3, 2009, pp. 199-203. doi:10.1016/j.autrev.2008.07.044

[28] J. H. Lee, T. Katakai, T. Hara, H. Gonda, M. Sugai and A. Shimizu, "Roles of p-ERM and Rho-ROCK Signaling in Lymphocyte Polarity and Uropod Formation," Journal of Cell Biology, Vol. 167, No. 2, 2004, pp. 327-337. doi:10.1083/jcb.200403091

[29] R. Nijhara, P. B. van Hennik, M. L. Gignac, M. J. Kruhlak, P. L. Hordijk, J. Delon, et al., "Rac1 Mediates Col- lapse of Microvilli on Chemokine-Activated T Lymphocytes,” Journal of Immunology, Vol. 173, No. 8, 2004, pp. 4985-4993.

[30] A. Delaguillaumie, C. Lagaudrière-Gesbert, M. R. Popoff and H. Conjeaud, "Rho GTPases Link Cytoskeletal Rearrangements and Activation Processes Induced Via the Tetraspanin CD82 in T Lymphocytes,” Journal of Cell Sciences, Vol. 115, No. 2, 2002, pp. 433-443.

[31] A. P. Wheelera and A. J. Ridley, "Why Three Rho Proteins? RhoA, RhoB, RhoC, and Cell Motility," Experimental Cell Research, Vol. 301, No. 1, 2004, pp. 43-49. doi:10.1016/j.yexcr.2004.08.012

[32] P. Tharaux, R. Bukoski, P. Rocha, S. Crowley, P. Ruiz, C. Nataraj, et al., "Rho Kinase Promotes Alloimmune Responses by Regulating the Proliferation and Structure of T Cells,” Journal of Immunology, Vol. 171, No. 1, 2003, pp. 96-105.

[33] S. Ohki, K. Iizuka, S. Ishikawa, M. Kano, K. Dobashi, A. Yoshii, et al., "A Highly Selective Inhibitor of Rho-Associated Coiled-Coil Forming Protein Kinase, Y-27632, Prolongs Cardiac Allograft Survival of the BALB/c-toC3H/He Mouse Model," Journal of Heart and Lung Transplantation, Vol. 20, No. 9, 2001, pp. 956-963. doi:10.1016/S1053-2498(01)00292-3 